\title{
ON THE NUMERICAL SOLUTION OF THE INITIAL-BOUNDARY VALUE PROBLEM WITH NEUMANN CONDITION FOR THE WAVE EQUATION BY THE USE OF THE LAGUERRE TRANSFORM AND BOUNDARY ELEMENTS METHOD
}

\author{
Svyatoslav LITYNSKYY*, Yuriy MUZYCHUK*, Anatoliy MUZYCHUK* \\ *Applied Mathematics and Informatics, Programming Department, Ivan Franko National University of Lviv, Universytetska 1, Lviv, Ukraine \\ s.litynskyy@gmail.com, yuriy.muzychuk@gmail.com, anatol.muzychuk@gmail.com
}

received 29 June 2016, revised 28 November 2016, accepted 1 December 2016

\begin{abstract}
Abstract. We consider a numerical solution of the initial-boundary value problem for the homogeneous wave equation with the Neumann condition using the retarded double layer potential. For solving an equivalent time-dependent integral equation we combine the Laguerre transform (LT) in the time domain with the boundary elements method. After LT we obtain a sequence of boundary integral equations with the same integral operator and functions in right-hand side which are determined recurrently. An error analysis for the numerical solution in accordance with the parameter of boundary discretization is performed. The proposed approach is demonstrated on the numerical solution of the model problem in unbounded three-dimensional spatial domain.
\end{abstract}

Key words: Boundary Integral Equation, Time-Domain Boundary Integral Equation, Wave Equation, Sobolev Spaces, Generalized Solution, Retarded Surface Potentials, Laguerre Transform, Boundary Elements Method

\section{INTRODUCTION}

The development of efficient numerical methods for initialboundary value problems for the wave equation in unbounded three-dimension spatial domains is actual for simulation of wave processes. In this formulation, approaches based on retarded potentials are widely used since they reduce the time-dependent problem in the unbounded domain to an integral equation on the bounded surface. Theory of such time-domain boundary integral equations (TDBIEs) for the wave equation was first developed by Bamberger and Ha-Duong (1986a, 1986b). In these papers the Galerkin method for TDBIEs was also proved. However, practical usage of the Galerkin method has some computational complexity, caused by the presence of dependency of potential density on the time and the spatial coordinates (so-called delay). It requires very precise spatial quadrature on parts of boundary elements which depend on delay, see, for example, Ha-Duong (2003) and Mykhas'kiv et al. (2014).

To overcome such difficulties after traditional discretizations on spatial variables some auxiliary problems may be considered for taking into account the time dependency. In particular, a convolution quadrature method (Lubich, 1994) has been utilized in many applications (see, for example, Laliena and Sayas, 2009; Monegato et al., 2011; Sayas and Qiu, 2015, and references there). It is based on sustainable methods for ordinary differential equations and is more efficient than Galerkin or collocation time approximations.

Another method is to apply the Fourier-Laplace integral transform with respect to the time variable as proposed in Bamberger and Ha-Duong (1986a), Ha-Duong (2003) and in Dominguez and Sayas (2013). This method is well suitable for theoretical investigations, however, the corresponding numerical inverse operation is complicated (except for some cases). In this respect the Laguerre transform $(L T)$, for which the inverse transform is to find the sum of corresponding Fourier-Laguerre serie, proved to be more constructive. In combination with the method of boundary integral equations (BIEs) such transform was used in papers Halazyuk et al. (1984), Jung et al. (2010), Litynskyy and Muzychuk (2015a), Lyudkevych and Muzychuk (1990), Chapko and Johansson (2016), Vavrychuk (2011) for numerical solution of various evolution problems.

In Litynskyy and Muzychuk (2015b, 2016) generalized solutions of the Dirichlet and Neumann initial-boundary value problems for the wave equation with homogeneous initial conditions were built by using the retarded potentials in some weighted Sobolev spaces, where desired solutions and densities of potentials allow the LT with respect to the time variable. Using this transform the Dirichlet and Neumann problems were reduced to sequences of BIEs. This work is dedicated to applying of the boundary elements method (BEM) to obtained equations in the case of the Neumann problem.

We begin in Section 2 with defining the Neumann initialboundary value problem for the wave equation and then we reduce it to an infinite triangular system of BIEs using LT. In Section 3 we rewrite it as a sequence of equations with the same integral operator in the left-hand side and apply the BEM to it with error analysis. At the end we consider results of the numerical experiment on solving of the model problem.

\section{REDUCTION OF THE NEUMANN PROBLEM TO AN INFINITE SYSTEM OF BIES}

Let $\Omega^{-}$be a domain in $\mathbb{R}^{3}$ with Lipschitz boundary $\Gamma, \Omega:=$ $\mathbb{R}^{3} \backslash \overline{\Omega^{-}}, \mathbb{R}_{+}:=(0, \infty), Q:=\Omega \times \mathbb{R}_{+}, \Sigma:=\Gamma \times \mathbb{R}_{+}$, and $\boldsymbol{v}(x)$ be a unit vector in the direction of the outward normal to the surface $\Gamma$ at a point $x \in \Gamma$.

We consider the initial-boundary value problem: find a function 
$u(x, t),(x, t) \in \bar{Q}$, that satisfies (in some sense) the homogeneous wave equation

$\frac{\partial^{2} u(x, t)}{\partial t^{2}}-\Delta u(x, t)=0, \quad(x, t) \in Q$,

homogeneous initial conditions

$u(x, 0)=0, \quad \frac{\partial u(x, 0)}{\partial t}=0, \quad x \in \Omega$,

and the Neumann boundary condition

$\partial_{\boldsymbol{v}(x)} u(x, t)=g(x, t), \quad(x, t) \in \Sigma$.

Here $\Delta$ is the Laplacian and $\partial_{v}$ denotes the normal derivative.

To solve the problem (1)-(3) we use the retarded double layer potential

$(\mathcal{D} \lambda)(x, t):=\left.\frac{1}{4 \pi} \int_{\Gamma} \partial_{\boldsymbol{v}(y)}\left(\frac{\lambda(z, t-|x-y|)}{|x-y|}\right)\right|_{z=y} d \Gamma_{y},(x, t) \in Q$,

where $\lambda: \Gamma \times \mathbb{R} \rightarrow \mathbb{R}$ is a density. It is known (see, e.g., Bamberger and Ha-Duong, 1986a or Polozhyy, 1964) that if an arbitrary function $\lambda(y, \tau),(y, \tau) \in \Gamma \times \mathbb{R}$, is smooth enough and $\lambda(y, \tau)=$ 0 when $y \in \Gamma, \tau \leq 0$, then function

$u(x, t):=(\mathcal{D} \lambda)(x, t), \quad(x, t) \in Q$,

satisfies (in the classical sense) the wave equation and initial conditions (2).

Note that for a sufficiently smooth density $\lambda$ and surface $\Gamma$ we can express the operator $\partial_{\boldsymbol{v}}$ as $\partial_{\boldsymbol{v}(x)} u(x, \cdot)=\boldsymbol{v}(x) \cdot \nabla_{x} u(x, \cdot)$, where $\nabla_{x}$ is the gradient operator. Then there exists the following limit

$(\mathcal{W} \lambda)(x, t):=$

$\left.\frac{1}{4 \pi} \boldsymbol{v}(x) \cdot \lim _{x^{\prime} \rightarrow x} \nabla_{x^{\prime}} \int_{\Gamma} \boldsymbol{v}(y) \cdot \nabla_{y}\left(\frac{\lambda\left(z, t-\left|x^{\prime}-y\right|\right)}{\left|x^{\prime}-y\right|}\right)\right|_{z=y} d \Gamma_{y}$,

where $x^{\prime}:=x-\varepsilon \boldsymbol{v}(x) \in \Omega, \varepsilon>0$, denotes a point close to the point $x \in \Gamma$. We say that $x^{\prime}$ approaches $x, x^{\prime} \rightarrow x$, when $\varepsilon \rightarrow 0$. The function $u$ satisfies the boundary condition (3), if the function $\lambda$ is a solution of the retarded potential integral equation (RPIE)

$(\mathcal{W} \lambda)(x, t)=g(x, t),(x, t) \in \Sigma$.

We shall briefly give the essential notions of the Laguerre transform in the weighted Lebesgue space $L_{\sigma}^{2}\left(\mathbb{R}_{+} ; X\right)$ with some parameter $\sigma>0$ and weight $\rho_{\sigma}(t)=e^{-\sigma t}, t \in \mathbb{R}_{+}$. Here $X$ is a Hilbert space with an inner product $(\cdot, \cdot)_{X}$ and an inducted norm $\|\cdot\|_{X}$. Elements $v \in L_{\sigma}^{2}\left(\mathbb{R}_{+} ; X\right)$ are measurable functions $v: \mathbb{R}_{+} \rightarrow X$ such that $\int_{\mathbb{R}_{+}}\|v(t)\|_{X}^{2} e^{-\sigma t} d t<\infty$. This space is equipped with the inner product

$(v, w)_{L_{\sigma}^{2}\left(\mathbb{R}_{+} ; X\right)}:=\int_{\mathbb{R}_{+}}(v(t), w(t))_{X} e^{-\sigma t} d t, v, w \in L_{\sigma}^{2}\left(\mathbb{R}_{+} ; X\right)$,

and the norm

$\|v\|_{L_{\sigma}^{2}\left(\mathbb{R}_{+} ; X\right)}:=\sqrt{(v, v)_{L_{\sigma}^{2}\left(\mathbb{R}_{+} ; X\right)}}, \quad v \in L_{\sigma}^{2}\left(\mathbb{R}_{+} ; X\right)$.

It is well-known (Reed and Simon, 1977) that the space $L_{\sigma}^{2}\left(\mathbb{R}_{+} ; X\right)$ is complete. We will assume that the elements of space $L_{\sigma}^{2}\left(\mathbb{R}_{+} ; X\right)$ are extended with zero for non-positive arguments.

For any $m \in \mathbb{N}$ (set of natural numbers) let us denote the weighted Sobolev space as

$H_{\sigma}^{m}\left(\mathbb{R}_{+} ; X\right):=\left\{v \in L_{\sigma}^{2}\left(\mathbb{R}_{+} ; X\right) \mid v^{(k)} \in L_{\sigma}^{2}\left(\mathbb{R}_{+} ; X\right), k=\overline{1, m}\right\}$ with the norm $\|v\|_{H_{\sigma}^{m}\left(\mathbb{R}_{+} ; X\right)}:=\left(\sum_{k=0}^{m}\left\|v^{(k)}\right\|_{L_{\sigma}^{2}\left(\mathbb{R}_{+} ; X\right)}^{2}\right)^{1 / 2}$. Here derivatives $v^{k}(k \in \mathbb{N})$ are understood in terms of the space $\mathcal{D}^{\prime}\left(\mathbb{R}_{+} ; X\right)$, elements of which are distributions with values in the space $X$.

We introduce a couple of notations. As the sequence of elements of set $X$ we understand a vector-column $v:=\left(v_{0}, v_{1}, \ldots\right)^{T}$. All possible sequences of elements of the set $X$ are denoted by $X^{\infty}$. We consider the Hilbert space $l^{2}(X):=\left\{\boldsymbol{v} \in X^{\infty} \mid \quad \sum_{j=0}^{\infty}\left\|v_{j}\right\|_{X}^{2}<+\infty\right\}$ with the inner product $(\boldsymbol{v}, \boldsymbol{w})=\sum_{j=0}^{\infty}\left(v_{j}, w_{j}\right)_{X}, \boldsymbol{v}, \boldsymbol{w} \in l^{2}(X)$, and the norm $\|\boldsymbol{v}\|_{l^{2}(X)}:=$ $\left(\sum_{j=0}^{\infty}\left\|v_{j}\right\|_{X}^{2}\right)^{1 / 2}, v \in l^{2}(X)$. Recall that for $X=\mathbb{R}$ we have $l^{2}(\mathbb{R})=l^{2}:=\left\{\left.\boldsymbol{v} \in \mathbb{R}^{\infty}\left|\quad \sum_{j=0}^{\infty}\right| v_{j}\right|^{2}<+\infty\right\}$.

Now let us give the definition of the Laguerre transform and outline some of its properties (Litynskyy and Muzychuk, 2015b). Consider a mapping $\mathcal{L}: L_{\sigma}^{2}\left(\mathbb{R}_{+} ; X\right) \rightarrow X^{\infty}$ which operates according to the rule

$f_{k}:=\sigma \int_{\mathbb{R}_{+}} f(t) L_{k}(\sigma t) e^{-\sigma t} d t, \quad k \in \mathbb{N}_{0}:=\mathbb{N} \cup\{0\}$,

where $\left\{L_{k}(\sigma \cdot)\right\}_{k \in \mathbb{N}_{0}}$ are Laguerre polynomials, which form orthogonal basis in the space $L_{\sigma}^{2}\left(\mathbb{R}_{+}\right)$(Keilson et al., 1980). Also consider the mapping $\mathcal{L}^{-1}: l^{2}(X) \rightarrow L_{\sigma}^{2}\left(\mathbb{R}_{+} ; X\right)$, which maps an arbitrary sequence $\boldsymbol{h}=\left(h_{0}, h_{1}, \ldots, h_{k}, \ldots\right)^{T}$ to a function

$h(t):=\left(\mathcal{L}^{-1} \boldsymbol{h}\right)(t)=\sum_{k=0}^{\infty} h_{k} L_{k}(\sigma t), t \in \mathbb{R}_{+}$.

Proposition 2.1 (theorem 2 from Litynskyy and Muzychuk, 2015b) The mapping $\mathcal{L}: L_{\sigma}^{2}\left(\mathbb{R}_{+} ; X\right) \rightarrow X^{\infty}$ that maps the arbitrary function $f$ to the sequence $\boldsymbol{f}=\left(f_{0}, f_{1}, \ldots, f_{k}, \ldots\right)^{T}$ according to the formula(10), is injective and its image is the space $l^{2}(X)$, and

$\|f\|_{L_{\sigma}^{2}\left(\mathbb{R}_{+} ; X\right)}^{2}=\frac{1}{\sigma} \sum_{k=0}^{\infty}\left\|f_{k}\right\|_{X}^{2}$.

In addition, for the arbitrary function $f \in L_{\sigma}^{2}\left(\mathbb{R}_{+} ; X\right)$ we have an equality

$\mathcal{L}^{-1} \mathcal{L} f=f$,

where the mapping $\mathcal{L}^{-1}: l^{2}(X) \rightarrow L_{\sigma}^{2}\left(\mathbb{R}_{+} ; X\right)$ is the inverse to $\mathcal{L}$ and maps the arbitrary sequence $\boldsymbol{h}=\left(h_{0}, h_{1}, \ldots, h_{k}, \ldots\right)^{T}$ to the function $h$ according to the formula (11).

Definition 2.2 (Litynskyy and Muzychuk, 2015b) Let $\sigma>0$ and $X$ be a Hilbert space. Mappings $\mathcal{L}: L_{\sigma}^{2}\left(\mathbb{R}_{+} ; X\right) \rightarrow$ $l^{2}(X)$ and $\mathcal{L}^{-1}: l^{2}(X) \rightarrow L_{\sigma}^{2}\left(\mathbb{R}_{+} ; X\right)$, mentioned in Proposition 2.1, are called, respectively, direct and inverse Laguerre transforms, and the formula (12) is an analogue of the Parseval equality.

Definition 2.3 (Litynskyy et al., 2009) Let $X, Y, Z$ be arbitrary sets and $q: X \times Y \rightarrow Z$ be some mapping. By a q-convolution of sequences $\boldsymbol{u} \in X^{\infty}$ and $\boldsymbol{v} \in Y^{\infty}$ we understand the sequence $\boldsymbol{w}:=\left(w_{0}, w_{1}, \ldots, w_{j}, \ldots\right)^{T} \in Z^{\infty}$, whose elements are obtained by the rule

$w_{j}:=\sum_{i=0}^{j} q\left(u_{j-i}, v_{i}\right) \equiv \sum_{i=0}^{j} q\left(u_{i}, v_{j-i}\right), \quad j \in \mathbb{N}_{0}$

the q-convolution of $\boldsymbol{u}$ and $\boldsymbol{v}$ is shortly written in the form $\boldsymbol{w}=$ $\boldsymbol{u} \circ \boldsymbol{v}$.

If $X=\mathcal{L}(Y, Z)$ is the space of linear operators acting from the space $Y$ into the space $Z$ and $q(A, v):=A v, A \in \mathcal{L}(Y, Z), v \in Y$, then for components of the q-convolution of arbitrary sequences 
$\boldsymbol{A} \in(\mathcal{L}(Y, Z))^{\infty}$ and $\boldsymbol{v} \in Y^{\infty}$ we will have the following formula

$w_{j}=\sum_{i=0}^{j} A_{j-i} v_{i}, \quad j \in \mathbb{N}_{0}$,

and will write $\boldsymbol{w}:=\boldsymbol{A}{ }_{Z} \boldsymbol{v}$.

Now let's consider a sequence of functions

$e_{0}(z):=\frac{e^{-\sigma|z|}}{4 \pi|z|}, \quad e_{k}(z):=\frac{e^{-\sigma|z|}}{4 \pi|z|}\left(L_{k}\left(\sigma|z|-L_{k-1}(\sigma|z|)\right), \quad k \in \mathbb{N}\right.$, $z \in \mathbb{R}^{3} \backslash\{0\}$.

Based on the above, in the space $H^{1}(\Omega):=\left\{v \in L^{2}(\Omega)|| \nabla v \mid \in\right.$ $\left.L^{2}(\Omega)\right\}$ we can define a function sequence

$\boldsymbol{u}(x):=\left(\boldsymbol{D}_{H^{1}(\Omega)}^{\circ} \lambda\right)(x), \quad x \in \Omega$,

where $\lambda=\mathcal{L} \lambda$ for any $\lambda \in L_{\sigma}^{2}\left(\mathbb{R}_{+} ; H^{1 / 2}(\Gamma)\right)$ and the sequence $\boldsymbol{D}$ is composed of operators $D_{k}: H^{1 / 2}(\Gamma) \rightarrow H^{1}(\Omega, \Delta), k \in \mathbb{N}_{0}$, given by the rule

$\left(D_{k} \xi\right)(x):=\frac{1}{4 \pi} \int_{\Gamma} \xi(y) \boldsymbol{v}(y) \cdot \nabla_{y} e_{k}(x-y) d \Gamma_{y}$.

Here $H^{1}(\Omega, \Delta):=\left\{v \in H^{1}(\Omega) \mid \Delta v \in L^{2}(\Omega)\right\}, H^{1 / 2}(\Gamma)$ denotes a space of traces of elements of $H^{1}(\Omega)$ on the surface $\Gamma$ and $H^{-1 / 2}(\Gamma):=\left(H^{1 / 2}(\Gamma)\right)^{\prime}$. If $u$ is expressed by the retarded double layer potential (4) with some density $\lambda$ then the sequence (17) represents the transformation $\boldsymbol{u}=\mathcal{L} u$ (Litynskyy and Muzychuk, 2016). Similarly, applying the LT to the equation (7), we obtain a BIE system

$\boldsymbol{W} \underset{H^{-1 / 2}(\Gamma)}{\circ} \lambda=\boldsymbol{g}$ in $l^{2}\left(H^{-1 / 2}(\Gamma)\right)$,

where $\boldsymbol{g}=\mathcal{L} g$ and $\boldsymbol{W}: l^{2}\left(H^{1 / 2}(\Gamma)\right) \rightarrow l^{2}\left(H^{-1 / 2}(\Gamma)\right)$ is a sequence of boundary operators

$\left(W_{k} \xi\right)(x):=\frac{1}{4 \pi} \boldsymbol{v}(x) \cdot \lim _{x^{\prime} \rightarrow x} \nabla_{x^{\prime}} \int_{\Gamma} \xi(y) \boldsymbol{v}(y) \cdot \nabla_{y} e_{k}\left(x^{\prime}-y\right) d \Gamma_{y}$,

$k \in \mathbb{N}_{0}$.

After finding the solution $\lambda=\left(\lambda_{0}, \lambda_{1}, \ldots\right)^{T}$ of the BIEs (19), the generalized solution of the problem (1)-(3) can be presented as a sum of the series

$u(x, t)=\sum_{k=0}^{\infty}\left(\sum_{i=0}^{k} D_{k-i} \lambda_{i}(x)\right) L_{k}(\sigma t), \quad(x, t) \in Q$.

Proposition 2.4 (theorem 2.4 from Litynskyy and Muzychuk, 2016) Let $g \in H_{\sigma_{0}}^{m+4}\left(\mathbb{R}_{+} ; H^{-1 / 2}(\Gamma)\right)$ for some $\sigma_{0}>0$ and $m \in \mathbb{N}_{0}$. Then there exists a unique generalized solution of the problem (1)-(3), it belongs to the space $H_{\sigma_{0}}^{m+1}\left(\mathbb{R}_{+} ; H^{1}(\Omega)\right)$ and for any $\sigma \geq \sigma_{0}$ such an inequality holds

$\|u\|_{H_{\sigma}^{m+1}\left(\mathbb{R}_{+} ; H^{1}(\Omega)\right)} \leq C\|g\|_{H_{\sigma}^{m+4}\left(\mathbb{R}_{+} ; H^{-1 / 2}(\Gamma)\right)}$,

where $C>0$ is a constant that is not dependent on $g$.

In addition, the generalized solution of the problem (1)-(3) can be obtained by the inverse transform $u=\mathcal{L}^{-1} \boldsymbol{u}$, where $u_{j} \in$ $H^{1}(\Omega, \Delta)\left(j \in \mathbb{N}_{0}\right)$ are the corresponding components of the $q$ convolution (17), and elements of the sequence $\lambda \in l^{2}\left(H^{1 / 2}(\Gamma)\right)$ are solutions of BIE system (19), in which $\boldsymbol{g}=\mathcal{L} g$.

\section{BEM FOR THE INFINITE BIE SYSTEM}

We have now the new representation (21) of the solution of the problem (1)-(3) and the infinite BIE system (19) with unknown functions $\lambda=\left(\lambda_{0}, \lambda_{1}, \ldots\right)^{T}$. It is easy to see that the sys- tem (19) can be rewritten as a sequence of BIEs

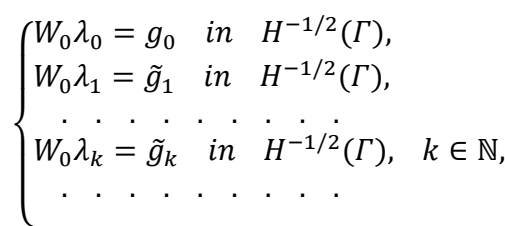

with recursive right-hand sides

$\tilde{g}_{k}:=g_{k}-\sum_{i=0}^{k-1} W_{k-i} \lambda_{i}, \quad k \in \mathbb{N}$.

For every $k \in \mathbb{N}_{0}$ the corresponding $k$-th equation of (23) is a hypersingular equation that has the form

$W_{0} \eta=f$ in $H^{-1 / 2}(\Gamma)$.

It has a unique solution $\eta \in H^{1 / 2}(\Gamma)$ for an arbitrary function $f \in H^{-1 / 2}(\Gamma)$ (Hsiao and Wendland, 2008). We can choose (by some criteria) value $N$ and find from (23) the first components $\lambda_{0}, \lambda_{1}, \ldots, \lambda_{N}$. Then the approximate solution of the problem (1)(3) is the partial sum

$u^{N}(x, t)=\sum_{j=0}^{N}\left(\sum_{i=0}^{k} D_{k-i} \lambda_{i}(x)\right) L_{k}(\sigma t), \quad(x, t) \in Q$.

We are now in position to apply the BEM for finding unknown functions. Consider in $H^{1 / 2}(\Gamma)$ a sequence of finite-dimensional subspaces $X^{M} \subset H^{1 / 2}(\Gamma), M \in N$, assuming that $\left\{\phi_{i}\right\}_{i=1}^{M}$ is a basis of $X^{M}$. The numerical solution of the equation (25) can be presented as a linear combination

$\eta^{M}:=\sum_{i=1}^{M} \eta_{i} \phi_{i} \in X^{M}$

that is a solution of such variational equation

$\left\langle W_{0} \eta^{M}, \eta\right\rangle_{\Gamma}=\langle f, \eta\rangle_{\Gamma} \quad \forall \eta \in X^{M}$.

Applying the Galerkin method, that is taking the elements of the basis $\phi_{j}$ as test functions in order to find the vector of unknown coefficients $\boldsymbol{\eta}^{[M]}:=\left\{\eta_{i}\right\}_{i=1}^{M} \in \mathbb{R}^{M}$, we will get a system of linear algebraic equations (SLAE)

$W_{0}^{[M]} \boldsymbol{\eta}^{[M]}=\boldsymbol{f}^{[M]}$.

where $W_{0}^{[M]}[j, i]:=\left\langle W_{0} \phi_{i}, \phi_{j}\right\rangle_{\Gamma}, f_{j}^{[M]}:=\left\langle f, \phi_{j}\right\rangle_{\Gamma}, \quad i, j=\overline{1, M}$. As a result of the $H^{1 / 2}(\Gamma)$-ellipticity of the operator $W_{0}$, the matrix $W_{0}^{[M]}$ is positive definite (Costabel M., 1988; Hsiao and Wedland, 1977; Schtainbah, 2008). Therefore, the system (29) has a unique solution for an arbitrary right-hand side $\forall M \in N$.

Let $\Gamma_{\widetilde{M}}=\bigcup_{l=1}^{\widetilde{M}} \bar{\tau}_{l}$ be some approximation of the boundary $\Gamma$ composed of triangular boundary elements $\left\{\tau_{l}\right\}_{l=1}^{\widetilde{M}}$ with vertices $\left\{x^{\left[l_{1}\right]}, x^{\left[l_{2}\right]}, x^{\left[l_{3}\right]}\right\}$. We assume that vertices of all triangles have a global numbering $\left\{x_{k}\right\}_{k=1}^{M^{*}}$ and for each point $x_{k}$ there exists an associated set $\mathcal{J}(k)$ of numbers of those triangles that have this point as a vertex. We treat the value $h:=\max _{l=1, \widetilde{M}}\left(\int_{\tau_{l}} \mathrm{~d} s\right)^{1 / 2}$ as the parameter of the spatial approximation.

Note that each triangle can be projected on a "standard"' triangle $\tau:=\left\{\xi:=\left(\xi_{1}, \xi_{2}\right) \in R^{2}: 0<\xi_{1}<1,0<\xi_{2}<1-\xi_{1}\right\}$. Following Dautray and Lions (1992) and Schtainbah (2008), functions $\phi_{1}^{1}(\xi)=1-\xi_{1}-\xi_{2}, \phi_{2}^{1}(\xi)=\xi_{1}$ and $\phi_{3}^{1}(\xi)=\xi_{2}$, defined locally on the triangle $\tau$, form a set $\left\{\varphi_{i}^{1}\right\}_{i=1}^{M}, M=M^{*}$, that contains linearly-independent on $\Gamma_{\widetilde{M}}$ functions. Moreover, $\operatorname{supp} \varphi_{i}^{1}=$ $\bigcup_{l \in \mathcal{J}(i)} \bar{\tau}_{l}=: \tau_{i}^{*}$. Then the numerical solution $\lambda^{h}:=\left(\lambda_{0}^{h}, \lambda_{1}^{h}, \ldots\right)^{T}$ of the BIE system (23) is sought in the form

$\lambda_{k}^{h}=\sum_{l=1}^{M} \lambda_{k, l}^{h} \varphi_{l}^{1} \quad \in S_{h}^{1}(\Gamma):=\operatorname{span}\left\{\varphi_{i}^{1}\right\}_{i=1}^{M}, k \in N_{0}$, 
where $\left\{\lambda_{k, l}^{h}\right\}_{l=1}^{M}$ are the unknown coefficients. In order to find the vector $\lambda_{k}^{h}:=\left\{\lambda_{k, l}^{h}\right\}_{l=1}^{M} \in \mathbb{R}^{M}$ we obtain the following SLAE from (29)

$\boldsymbol{W}_{0}^{h} \lambda_{k}^{h}=\boldsymbol{g}_{k}^{h}-\sum_{i=0}^{k-1} \boldsymbol{W}_{k-i}^{h} \lambda_{i}^{h}, \quad k \in N$,

where

$W_{j}^{h}[i, l]=\int_{\tau_{i}^{*}} \varphi_{i}^{1}(x) \partial_{v(x)} \int_{\tau_{l}^{*}} \varphi_{l}^{1}(y) \partial_{v(y)} E_{j}(x-y) d s_{y} d s_{x}, j=$

$\overline{0, k}$,

$g_{k}^{h}[i]=\int_{\tau_{i}^{*}} \varphi_{i}^{1}(x) \tilde{g}_{k}(x) d s_{x}, \quad i, l=\overline{1, M}$.

After finding the consequent vector $\lambda_{\mathrm{k}}^{\mathrm{h}}$ we can compute the corresponding element of the sequence $\boldsymbol{u}^{h}:=\left(u_{0}^{h}, u_{1}^{h}, \ldots, u_{k}^{h}, \ldots, u_{N}^{h}, 0, \ldots\right)^{T}$ :

$u_{k}^{h}(x)=\sum_{j=0}^{k}\left(D_{j} \lambda_{k-j}^{h}\right)(x), x \in \Omega$.

Having $\boldsymbol{u}^{h}$, the numerical solution of the Neumann problem is found by the formula

$u^{N, h}(x, t)=\sum_{j=0}^{N} u_{k}^{h}(x) L_{k}(\sigma t), \quad(x, t) \in Q$.

Let us obtain, following Hsiao and Wedland (1977), an a priory error estimate of the numerical solution after the introduction of Sobolev spaces of piecewise-smooth on the boundary $\Gamma$ functions. Let $\Gamma$ be a union $\Gamma=\bigcup_{i=1}^{\widetilde{N}} \bar{\Gamma}_{i}$ of surfaces $\Gamma_{i}\left(\Gamma_{i} \cap \Gamma_{j}=\varnothing\right.$ when $i \neq j$ ), each of which has a sufficiently smooth parameterization $\Gamma_{i}:=\left\{x \in R^{3}: x=\tilde{\chi}_{i}(\xi), \xi \in \tilde{\tau}_{i} \subset R^{2}\right\}$. Then, using the set of the non-negative functions $\phi_{i} \in C_{0}^{\infty}\left(R^{3}\right)$ such that $\sum_{i=1}^{\widetilde{N}} \phi_{i}(x)=1 \quad \forall x \in \Gamma, \quad \phi_{i}(x)=0 \quad \forall x \in \Gamma \backslash \Gamma_{i}$,

an arbitrary piecewise-smooth function can be given in a form

$v(x)=\sum_{i=1}^{\widetilde{N}} \phi_{i}(x) v(x)=\sum_{i=1}^{\widetilde{N}} v_{i}(x) \quad \forall x \in \Gamma$,

where $v_{i}(x):=\phi_{i}(x) v(x) \quad \forall x \in \Gamma_{i}$. Taking into account the parameterization of the fragments $\Gamma_{i}$, we consider Sobolev spaces $H^{s}\left(\tilde{\tau}_{i}\right)$, elements of which are functions $\tilde{v}_{i}(\xi):=v_{i}\left(\tilde{x}_{i}(\xi)\right)$ when $\xi \in \tilde{\tau}_{i}$, with corresponding norms and semi-norms

$\left\|\tilde{v}_{i}\right\|_{H^{s}\left(\tilde{\tau}_{i}\right)}:=\left(\sum_{|\alpha| \leq m}\left\|\partial^{\alpha} \tilde{v}_{i}\right\|_{L^{2}\left(\tilde{\tau}_{i}\right.}^{2}\right)^{\frac{1}{2}}$

$\left|\tilde{v}_{i}\right|_{H^{s}\left(\tilde{\tau}_{i}\right)}:=\left(\sum_{|\alpha|=m}\left|\partial^{\alpha} \tilde{v}_{i}\right|_{L^{2}\left(\tilde{\tau}_{i}\right)}^{2}\right)^{\frac{1}{2}}, \quad s=m \in N$;

$\left|\tilde{v}_{i}\right|_{H^{s}\left(\tilde{\tau}_{i}\right)}:=\left(\sum_{|\alpha|=m} \int_{\tilde{\tau}_{i}} \int_{\tilde{\tau}_{i}} \frac{\left|\partial^{\alpha} \tilde{v}_{i}(\xi)-\partial^{\alpha} \tilde{v}_{i}(\eta)\right|^{2}}{|\xi-\eta|^{2+2 \sigma}} d s_{\xi} d s_{\eta}\right)^{\frac{1}{2}}$,

$s=m+\sigma, \sigma \in(0,1)$.

Here $\partial^{\alpha}$ is a notation of the partial derivative with a multi-index $\alpha=\left(\alpha_{1}, \alpha_{2}\right)$. Moreover, for functions that are defined on the whole boundary $\Gamma$, we use the semi-norm

$|v|_{H^{s}(\Gamma)}:=\left(\sum_{i=1}^{\widetilde{N}}\left|\tilde{v}_{i}\right|_{H^{s}\left(\tilde{\tau}_{i}\right)}^{2}\right)^{1 / 2}$.

Lemma 3.1 Let $\lambda \in\left(H^{s}(\Gamma)\right)^{\infty}$ for some $s \in\left[\frac{1}{2}, 2\right]$ be the exact solution of the BIE system (23), which satisfies an inequality

$\sum_{j=0}^{\infty}\left|\lambda_{j}\right|_{H^{s}(\Gamma)}<+\infty$.

Then for any values of parameters $N \in \mathbb{N}_{0}$ and $T \in \mathbb{R}_{+}$the following error estimates hold

$\left\|\lambda^{N}(\cdot, t)-\lambda^{N, h}(\cdot, t)\right\|_{H^{\frac{1}{2}(\Gamma)}} \leq \tilde{C}_{N, T} h^{s-\frac{1}{2}} \sum_{k=0}^{N}\left|\lambda_{k}\right|_{H^{s}(\Gamma)}$,

$t \in(0, T)$,
$\left|u^{N}(x, t)-u^{N, h}(x, t)\right| \leq \tilde{C}_{N, T}^{*} h^{s-\frac{1}{2}} \sum_{k=0}^{N}\left|\lambda_{k}\right|_{H^{s}(\Gamma)}$,

$x \in \Omega, \quad t \in(0, T)$,

where $\tilde{C}_{N, T}$ and $\tilde{C}_{N, T}^{*}$ are constants independent of $h$.

Proof. Let us fix two arbitrary values $N \in \mathbb{N}_{0}$ and $T \in \mathbb{R}_{+}$and consider $\delta_{N, T}:=\left\|\lambda^{N}(\cdot, t)-\lambda^{N, h}(\cdot, t)\right\|_{H^{1 / 2}(\Gamma)}=\| \sum_{k=0}^{N}\left(\lambda_{k}(\cdot)-\right.$ $\left.\lambda_{k}^{h}(\cdot)\right) L_{k}(\sigma t) \|_{H^{1 / 2}(\Gamma)} \quad$ for any $t \in(0, T)$. Setting $C_{N, T}:=$ $\max _{t \in \overline{0}, T], \overline{0}}\left|L_{k}(\sigma t)\right|$ we can write

$\delta_{N, T} \leq C_{N, T} \sum_{k=0}^{N}\left\|\lambda_{k}-\lambda_{k}^{h}\right\|_{H^{1 / 2}(\Gamma)}$.

Note that for any function $\lambda_{k}$, which satisfies an equation like (25), the inequality (39) yields the following estimate (Schtainbah, 2008)

$\| \lambda_{k}-\left.\lambda_{k}^{h}\right|_{H^{1 / 2}(\Gamma)} \leq \tilde{C_{k}} h^{s-1 / 2}\left|\lambda_{k}\right|_{H^{s}(\Gamma)}, \quad k \in N_{0}$,

where $\tilde{C}_{k}$ are constants independent of $h$. Using this inequality and setting $\tilde{C}_{N, T}:=C_{N, T} \max _{k=0, N}\left\{\tilde{C}_{k}\right\}$ we obtain (40) from (42).

Since in the case of Lipschitz boundary all functions $E_{j}(x-$-) are bounded and infinitely continuously differentiable on $\Gamma$ for any fixed point $x \in \Omega$, we get inequality $\left\|\partial_{\nu(\cdot)} E_{j}(x-\cdot)\right\|_{H^{-1 / 2}(\Gamma)} \leq$ $c_{j}^{*}=$ const. Taking it and (43) into account by the Generalized Cauchy-Schwarz inequality we obtain $\left|u_{k}(x)-u_{k}^{h}(x)\right|=$ $\left|\sum_{i=0}^{k}\left\langle\partial_{\nu(\cdot)} E_{k-i}(x-\cdot),\left(\lambda_{i}-\lambda_{i}^{h}\right)\right\rangle_{\Gamma}\right| \leq \tilde{c}_{k} h^{s-1 / 2} \sum_{i=0}^{k}\left|\lambda_{i}\right|_{H^{s}(\Gamma)}$, where $\tilde{c}_{k}$ are constants independent of $h$. Using this estimate, the rest of the proof for (41) can be carried out analogously to the proof for (40).

\section{RESULTS OF THE COMPUTATIONAL EXPERIMENT}

Let us demonstrate the suggested method to solve some model problem and assess the accuracy of numerical solutions. Let the domain $\Omega:=R^{3} \backslash \Omega^{-}$be outside of a cube $\Omega^{-}:=[-1,1] \times$ $[-1,1] \times[-1,1]$ and the function $g(x, t):=-\partial_{v(x)} v(x, t)$, $(x, t) \in \Sigma$, in the boundary condition (3) is defined by means of a spherical impulse $v(x, t):=f_{3}(t-|x|+1)|x|^{-1},(x, t) \in Q$, where $f_{3}$ is a cubic $B$-spline.

Tab. 1. Convergence behavior of $u_{0}^{h}(x)$ at points $x=\left(x_{1}, 0,0\right)$

\begin{tabular}{|c|c|c|c|c|}
\hline & \multicolumn{3}{|c|}{$u_{0}^{h}(x)$} & \multirow[t]{2}{*}{$u_{0}(x)$} \\
\hline$x_{1}$ & $\bar{M}=588$ & $\bar{M}=1200$ & $\bar{M}=1728$ & \\
\hline 1.2 & $\begin{array}{l}4.97567 \\
\times 10^{-1}\end{array}$ & $\begin{array}{l}5.16241 \\
\times 10^{-1}\end{array}$ & $\begin{array}{l}5.23443 \\
\times 10^{-1}\end{array}$ & $\begin{array}{l}5.58600 \\
\times 10^{-1}\end{array}$ \\
\hline 1.5 & $\begin{array}{l}2.19226 \\
\times 10^{-1}\end{array}$ & $\begin{array}{l}2.26607 \\
\times 10^{-1}\end{array}$ & $\begin{array}{l}2.29583 \\
\times 10^{-1}\end{array}$ & $\begin{array}{l}2.45252 \\
\times 10^{-1}\end{array}$ \\
\hline 2.0 & $\begin{array}{l}6.07058 \\
\times 10^{-2}\end{array}$ & $\begin{array}{l}6.25240 \\
\times 10^{-2}\end{array}$ & $\begin{array}{l}6.32929 \\
\times 10^{-2}\end{array}$ & $\begin{array}{l}6.76676 \\
\times 10^{-2}\end{array}$ \\
\hline 3.0 & $\begin{array}{l}5.50191 \\
\times 10^{-3}\end{array}$ & $\begin{array}{l}5.64833 \\
\times 10^{-3}\end{array}$ & $\begin{array}{l}5.71311 \\
\times 10^{-3}\end{array}$ & $\begin{array}{l}6.10521 \\
\times 10^{-3}\end{array}$ \\
\hline 4.0 & $\begin{array}{l}5.59834 \\
\times 10^{-4}\end{array}$ & $\begin{array}{l}5.73967 \\
\times 10^{-4}\end{array}$ & $\begin{array}{l}5.80360 \\
\times 10^{-4}\end{array}$ & $\begin{array}{l}6.19688 \\
\times 10^{-4}\end{array}$ \\
\hline
\end{tabular}


Since the function $v$ satisfies the wave equation in $\Omega$ we can use it as a analytical solution of the Neumann problem (1)-(3). Following Muzychuk and Chapko (2012), we can also consider elements of sequence $\boldsymbol{v}(x)=\mathcal{L} v(x, \cdot), x \in \Omega$, as exact values for q-convolution $\boldsymbol{u}^{h}(x)$, computed according to the formula (34) on the solution $\lambda^{\mathrm{h}}$ of $\mathrm{BIE}(23)$ with $g_{k}=-\partial_{v} v_{k}, k \in \mathbb{N}_{0}$, in righthand side.

At first we consider how parameter $h$ of BEM, that depends on the number $\bar{M}$ of boundary elements on the surface $\Gamma$, has effect on the approximation error of elements of the sequence $v$ via corresponding elements of the sequence $\boldsymbol{u}^{h}$. Table 1 contains values of numerical solutions $u_{0}^{h}$ computed with various values $\bar{M}$. As expected, at all points of observation these values tend to corresponding exact values $u_{0}$ with decreasing of the value $h$. Note, that components $u_{k}^{h}, k \in \mathbb{N}$, have the same convergence behaviour.

Tab. 2. Error analysis of $u_{0}^{h}(x)$

\begin{tabular}{|c|c|c|c|}
\hline$\overline{\boldsymbol{M}}$ & $\boldsymbol{\delta}^{\boldsymbol{h}}$ & $\boldsymbol{e o c}$ & $\boldsymbol{\varepsilon}^{\boldsymbol{h}}(\%)$ \\
\hline 300 & 0.03539 & & 8.34 \\
\hline 588 & 0.02565 & 0.957 & 5.92 \\
\hline 768 & 0.02254 & 0.967 & 5.16 \\
\hline 972 & 0.02011 & 0.969 & 4.58 \\
\hline 1200 & 0.01815 & 0.985 & 4.11 \\
\hline 1728 & 0.01518 & 0.992 & 3.32 \\
\hline
\end{tabular}

Let us now compute values $\delta^{h}:=\left\|u_{0}^{h}-u_{0}\right\|_{L^{2}(a, b)}$ and $\varepsilon^{h}:=\delta^{h}\left\|u_{0}\right\|_{L^{2}(a, b)}^{-1} \cdot 100 \%$, where $(a, b)$ is a spatial interval from which observation points $x$ are taken, and also evaluate the estimated order of convergence eoc: $=\left(\ln \delta^{h_{j}}-\ln \delta^{h_{j+1}}\right)\left(\ln h_{j}-\right.$ $\left.\ln h_{j+1}\right)^{-1}$ (Schtainbah, 2008), where $h_{j}$ and $h_{j+1}$ are parameters of consequent spatial discretizations. Computed with $a=$ $(1.2,0,0)$ and $b=(10,0,0)$ results are given in Tab. 2. They highlight that $e o c \approx 1$ for this class of problems. Note, that an analogous behaviour is intrinsic also to $u_{k}^{h}, k \in \mathbb{N}$.

On Figure 1 numerical solutions $u^{h, N}(x, t)$ of the Neumann problem (1)-(3), computed with various values of the parameter $\mathrm{N}$ in the partial sum (35) and $\bar{M}=972$, are plotted as a comparison against the analytical solution $v(x, t)$. As we can see these results have close agreement in some initial time interval already for not large values $N$. This interval can be enlarged with increasing $N$. Numerical solutions $u^{h, N}(x, t)$ computed with $N=40$ and $\bar{M}=972$ are presented on Figure 2. They demonstrate signal propagation at different observation points $x$.

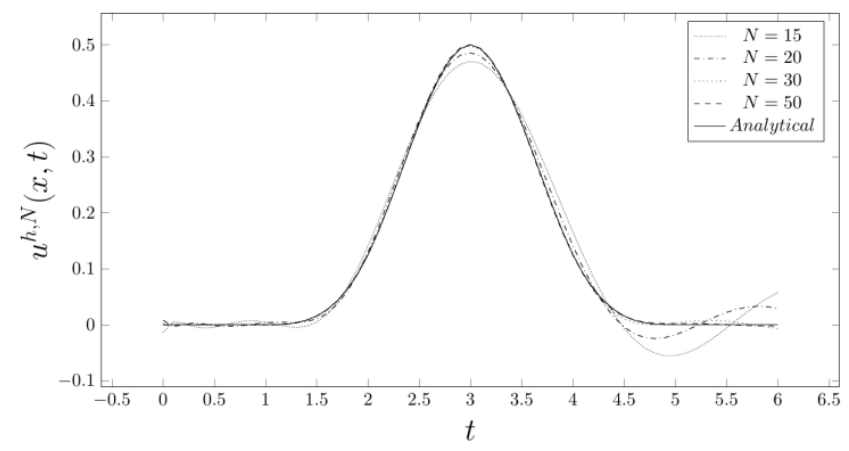

Fig.1. Numerical solutions $u^{h, N}(x, t)$ of problem (1)-(3) at point $x=(2,0,0)$ with various values $N$

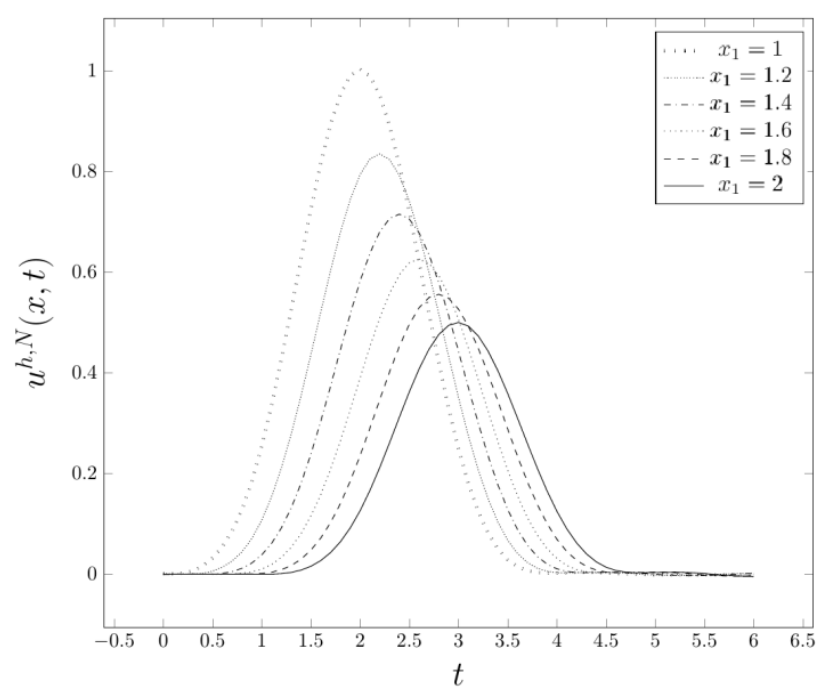

Fig. 2. Numerical solutions $u^{h, N}(x, t)$ of problem (1)-(3) at points $x=\left(x_{1}, 0,0\right)$

\section{CONCLUSION}

The proposed method enables us to find the numerical solution of the Neumann problem for the homogeneous wave equation with homogeneous initial conditions. Applying the LT, we omit the delay in the retarded double layer potential and in the time-domain BIE making computation process clearer. As result we obtain the sequence of BIEs which enable us to use the BEM efficiently.

Results of computational experiments confirm apriori error estimates that are obtained in the article.

\section{REFERENCES}

1. Bamberger A., Ha-Duong T. (1986a), Variational formulation for calculating the diffraction of an acoustic wave by a rigid surface, Math. Methods Appl. Sci., 8(4), 598-608 (in French).

2. Bamberger A., Ha-Duong T. (1986b), Variational space-time formulation for computation of the diffraction of an acoustic wave by the retarded potential (I), Math. Methods Appl. Sci., 8(3), 405435 (in French).

3. Chapko R., Johansson B. T. (2016), Numerical solution of the Dirichlet initial boundary value problem for the heat equation in exterior 3-dimensional domains using integral equations, Journal of Engineering Mathematics, 1-15.

4. Costabel M. (1988), Boundary integral operators on Lipschitz domains: elementary results, SIAM J. Math. Anal., V. 19, 613626.

5. Dautray R., Lions J. L. (1992), Mathematical analysis and numerical methods for science and technology, Volume 5 Evolution problems I., Springer-Verlag, Berlin.

6. Dominguez V., Sayas F. J. (2013), Some properties of layer potentials and boundary integral operators for wave equation, Journal of Integral Equations and Applications, 25(2), 253-294.

7. Ha-Duong T. (2003), On retarded potential boundary integral equations and their discretization, In Davies P.; Duncan D.; Martin P.; Rynne B. (eds.): Topics in computational wave propagation. Direct and inverse problems, Berlin: Springer-Verlag, 301-336.

8. Halazyuk V. A., Lyudkevych Y. V., Muzychuk A. O. (1984), Method of integral equations in non-stationary defraction problems, LSU. Dep. in UkrNIINTI, 601 (in Ukrainian).

9. Hsiao G. C., Wendland W. L. (1977), A finite element method for some integral equations of the first kind, J. Math. Anal. Appl., 58, 449-481. 
10. Hsiao G. C., Wendland W. L. (2008), Boundary Integral Equations, Applied Mathematical Sciences, Springer-Verlag Berlin Heidelberg.

11. Jung B. H., Sarkar T. K., Zhang Y., Ji Z., Yuan M., Salazar-Palma M., Rao S. M., Ting S. W., Mei Z., De A. (2010), Time and frequency domain solutions of em problems using integral equations and a Hybrid methodology, Wiley-IEEE Press.

12. Keilson J. (1981), The bilateral Laguerre transform, Applied Mathematics and Computation, 8(2), 137-174.

13. Laliena A. R., Sayas F. J. (2009), Theoretical aspects of the application of convolution quadrature to scattering of acoustic waves, Numer. Math., 112(4), 637-678.

14. Litynskyy S., Muzychuk A. (2015a), Retarded Potentials and Laguerre Transform for Initial-Boundary Value Problems for the Wave Equation, 20th IEEE International Seminar/Workshop on Direct and Inverse Problems of Electromagnetic and Acoustic Wave Theory (DIPED-2015), Lviv: Pidstryhach IAPMM of NASU, 139-142 (in Ukrainian).

15. Litynskyy S., Muzychuk A. (2015b), Solving of the initial-boundary value problems for the wave equation by the use of retarded potential and the Laguerre transform, Matematychni Studii, 44(2), 185-203 (in Ukrainian).

16. Litynskyy S., Muzychuk A. (2016), On the generalized solution of the initial-boundary value problems with Neumann condition for the wave equation by the use of retarded double layer potential and the Laguerre transform, Journal of Computational and Applied Mathematics, 2(122), 21-39.

17. Litynskyy S., Muzychuk Yu., Muzychuk A. (2009), On weak solutions of boundary problems for an infinite triangular system of elliptic equations, Visnyk of the Lviv university. Series of Applied mathematics and informatics, 15, 52-70 (in Ukrainian).

18. Lubich Ch. (1994), On the multistep time discretization of linear initial-boundary value problems and their boundary integral equations, Numer. Math., 365-389.
19. Lyudkevych Y. V., Muzychuk A. (1990), Numerical solution of boundary problems for wave eqution, L'viv: LSU, 80 (in Ukrainian).

20. Monegato G., Scuderi L., Stanic M. P. (2011), Lubich convolution quadratures and their application to problems described by spacetime BIEs, Numerical Algorithms, Springer Science, 3(56), 405-436.

21. Muzychuk Yu. A., Chapko R. S. (2012), On variational formulations of inner boundary value problems for infinite systems of elliptic equations of special kind, Matematychni Studii, 38(1), 12-34.

22. Mykhas'kiv V. V., Martin P. A., Kalynyak O. I. (2014), Timedomain BEM for 3-D transient elastodynamic problems with interacting rigid movable disc-shaped inclusions, Computational Mechanics, 53(6), 1311-1325.

23. Polozhyy H. (1964), Equations of mathematical physics, Nauka, Moscow (in Russian).

24. Reed M., Simon B. (1977), Methods of modern mathematical physics, Mir, Moscow (in Russian).

25. Sayas F. J., Qiu T. (2015), The Costabel-Stephan system of Boundary Integral Equations in the Time Domain, Mathematics of Computation, 85, 2341-2364.

26. Schtainbah 0. (2008), Numerical Approximation Methods for Elliptic Boundary Value Problems. Finite and Boundary Elements, Springer Science.

27. Vavrychuk V. H. (2011), Numerical solution of mixed non-stationary problem of thermal conductivity in partially unbounded domain, Visnyk of the Lviv university, Series of Applied mathematics and informatics, 17, $62-72$ (in Ukrainian).

The work has been accomplished under the research project at Ivan Franko National University of Lviv (State budgetary program "Numerical solution of linear and nonlinear problems of computational mathematics" \# 0110U003150). 